OPEN ACCESS

Edited by:

Leigh Breen,

University of Birmingham

United Kingdom

Reviewed by:

Theocharis Ispoglou, Leeds Beckett University,

United Kingdom

Darren Candow

University of Regina, Canada

${ }^{*}$ Correspondence:

Andrea R. Josse

ajosse@yorku.ca

Val A. Fajardo

vfajardo@brocku.ca

tThese authors have contributed

equally to this work

¥These authors have contributed equally to this work and share senior authorship

Specialty section:

This article was submitted to Sport and Exercise Nutrition,

a section of the journa

Frontiers in Nutrition

Received: 19 October 2021 Accepted: 23 November 2021

Published: 22 December 202

Citation:

Baranowski RW, Skelly LE, Josse AR and Fajardo VA (2021) Exploring the

Effects of Greek Yogurt

Supplementation and Exercise Training on Serum Lithium and Its Relationship With Musculoskeletal

Outcomes in Men.

Front. Nutr. 8:798036

doi: 10.3389/fnut.2021.798036

\section{Exploring the Effects of Greek Yogurt Supplementation and Exercise Training on Serum Lithium and Its Relationship With Musculoskeletal Outcomes in Men}

\author{
Ryan W. Baranowski ${ }^{1,2 \dagger}$, Lauren E. Skelly ${ }^{3 \dagger}$, Andrea R. Josse ${ }^{1,2,3 * \pm}$ and Val A. Fajardo ${ }^{1,2 * \neq}$ \\ ${ }^{1}$ Centre for Bone and Muscle Health, Brock University, St. Catharines, ON, Canada, ${ }^{2}$ Department of Kinesiology, Faculty of \\ Applied Health Sciences, Brock University, St. Catharines, ON, Canada, ${ }^{3}$ School of Kinesiology and Health Science, Faculty \\ of Health, York University, Toronto, ON, Canada
}

Dairy products can act as a dietary source of lithium (Li), and a recent study in universityaged males demonstrated that Greek yogurt (GY) supplementation augmented gains in fat free mass, strength and bone formation after 12 weeks of resistance exercise training compared to carbohydrate $(\mathrm{CHO})$ pudding supplementation. Here, we performed secondary analyses to explore whether GY would alter serum Li levels and whether changes in serum Li would associate with changes in body composition, strength, and bone turnover markers. Results show that the GY group maintained serum Li levels after exercise training, whereas the $\mathrm{CHO}$ group did not. Maintaining/elevating serum Li levels was also associated with greater gains in strength and reductions in bone resorption. However, controlling for other dietary factors in GY such as protein and calcium weakened these associations. Thus, future studies should assess the causative role, if any, of dietary Li alone on strength and bone resorption in humans.

Keywords: body composition, strength, bone turnover, dairy, resistance training, P1NP, CTX

\section{INTRODUCTION}

Lithium ( $\mathrm{Li}$ ) is a monovalent cation commonly prescribed for bipolar disorder; however, psychiatric use of Li requires a dose high enough $(\sim 0.8 \mathrm{mM}$ serum Li) to penetrate the blood brain barrier and to exert benefits on mental health (1). Unfortunately, these high doses of Li have been associated with adverse effects to peripheral organs such as the kidney, thyroid, and gastrointestinal tract (2-5). Conversely, studies in rodent models have shown that low dose Li supplementationwith levels well below those used for bipolar disorder-can provide several physiological benefits. For example, in 2010, Choi and colleagues showed that $10 \mathrm{mg} / \mathrm{kg} /$ day supplementation of $\mathrm{Li}$, which we have shown results in a serum [Li] of $0.02 \mathrm{mM}$ (6), attenuated high-fat diet induced obesity and atherosclerosis in mice (7). With this same dose, we have shown that 6-weeks of $\mathrm{Li}$ supplementation can enhance skeletal muscle force production and osteogenic signaling that favors bone formation over resorption $(6,8)$. In all, it is possible that the adverse effects associated with chronic use of high dose Li therapy may have led to an underappreciation of the benefits of low dose Li supplementation; however, whether these benefits translate to humans remains unknown. 
Aside from therapy, Li can be naturally obtained from the diet with main food sources including cereals, potatoes, tomatoes, cabbage, dairy products (i.e., milk and yogurt), and in some mineral- and tap-water (depending on geographical location) (9-12). In a recent study by Bridge et al. (13), Greek yogurt (GY) supplementation enhanced fat free mass (FFM) gains, biceps thickness, and total strength after a 12week resistance and plyometric exercise program in untrained, university-aged males (13). In addition, another analysis from this study discovered that GY supplementation increased bone formation demonstrated by an increased concentration of the bone formation marker procollagen type $1 \mathrm{~N}$-terminal propeptide (P1NP) and an increase in the ratio of P1NP to the bone resorption marker $\beta$-isomerized carboxy-terminal crosslinking telopeptides (CTX) in serum after the 12-week exercise intervention (14). Given that $\mathrm{Li}$ can be found in dairy products such as yogurt $(0.07 \pm 0.04 \mathrm{mg} / \mathrm{kg})$ (9), in this exploratory analysis we examined whether GY supplementation altered serum Li levels, and whether changes in serum Li were associated with various changes in body composition, strength, and markers of bone turnover after 12 weeks of exercise training in young males.

\section{MATERIALS AND METHODS}

\section{Participants, Ethics Approval, and Study Design}

Thirty healthy (free of medical conditions), young, untrained, adult (18-25 y) males were recruited from Brock University (Ontario, Canada) between July 2017 and August 2018 to participate in the Brock Exercise And Supplement Trial (BEAST) as previously described (13). All subjects were informed of potential study risks, and written informed consent was obtained. The original BEAST Study was approved by the Brock University Biosciences Research Ethics Board (REB\#16295) and registered at clinicaltrials.gov (NCT03196856), and these exploratory analyses were subsequently approved (REB\#20172).

This parallel, randomized, controlled, intervention study divided subjects into two groups: a Greek yogurt group (GY; $n=15)$ and a Placebo carbohydrate $(\mathrm{CHO})$ pudding group $(n$ $=15$ ). The GY group consumed $200 \mathrm{~g}$ of Oikos $0 \%$ fat, plain GY (110 Kcals, $20 \mathrm{~g}$ protein, $8 \mathrm{~g}$ CHO; Danone Canada Inc., Boucherville, Quebec) 3 times/day on training days (immediately post-exercise, 1 -h post-exercise, and before bed) and $150 \mathrm{~g} 2$ times/day on non-training days (breakfast and before bed). The CHO group consumed $47 \mathrm{~g}$ of an isoenergetic, chocolate flavored, CHO-based semi-solid pudding ( $110 \mathrm{Kcals}, 0 \mathrm{~g}$ protein, $28 \mathrm{~g} \mathrm{CHO}$ ) that was designed to resemble the consistency and texture of GY on the same schedule as the GY group. The CHO supplement's contents were kept discreet to participants. Many participants believed this supplement was the "test product" and that it may have contained muscle-supporting nutrients like protein. The 12-week exercise protocol consisted of supervised exercise training sessions 3 days/week. Sessions included fullbody resistance training at $70 \%$ of 1 -repetition maximum strength (2/d week) and plyometric training (1/d week). All participants were provided with the same information and advice to help them compensate for the added calories consumed from the supplements, as a means to avoid participants being in a positive energy balance. Additional details regarding the exercise training and supplementation program have been published elsewhere (13).

\section{Body Composition, Biceps Thickness, Strength, Markers of Bone Turnover, and Dietary Records}

Pre- and post-study measures of body composition (body mass, FFM, fat mass and \% body fat via Bodpod [Cosmed USA Inc. Chicago, Il]), biceps and quadriceps muscle thickness (via ultrasonography [GE Medical Systems, Ultrasound Vivid I portable, Milwaukee, WI, USA]), total strength (combined 1-repetition maximum scores of chest press, seated row, leg extension, and hamstring curl) and circulating levels of the bone turnover biomarkers P1NP (Roche Diagnostics; cat. no. 03141071190 via Roche Elecsys e411 automated analyzer) and CTX ( $\beta$-CrossLaps; cat. no.: 11972308122 via Roche Cobas e602 automated analyzer) were assessed as previously described $(13,14)$. Dietary calcium, protein, phosphorus and potassium intake were all measured using 7-day (at baseline) and 3-day (during the 12th week of training) food diaries (13).

\section{Serum Lithium Analysis}

Serum and GY Li concentrations were measured using inductively coupled plasma mass spectrometry (ICP-MS) as previously described (6).

\section{Statistics}

Baseline comparisons between $\mathrm{CHO}$ and GY were assessed using an independent $t$-test. Assumptions of normality for all data were tested using a D'Agostino \& Pearson test. A two-way repeated measures ANOVA, with a Bonferroni posthoc test was used to examine changes in serum Li pre- and post-exercise training in the GY and CHO groups. Pearson's or Spearman's correlational analyses were done, depending on tests for normality, to examine the association between absolute changes in serum Li and changes in body mass, body composition, muscle thickness, strength, and markers of bone turnover (change $=$ post-pre) using pooled $(\mathrm{GY}+\mathrm{CHO})$ data. Partial correlations were performed to control for change in components of dairy, including calcium, protein, phosphorus, and potassium. Analyses were only conducted in 25 of the total 30 participants as 2 did not have post-exercise blood samples (GY $n=1$; CHO $n=1$ ), 2 did not complete the full 12 weeks of training (GY $n=1$; $\mathrm{CHO} n=1$ ), and one did not provide a 3-day post-intervention food diary (GY $n=$ $1)$. Thus, from the original allocation of $n=15$ per group, we analyzed datasets from $12 \mathrm{GY}$ and $13 \mathrm{CHO}$ participants. Statistical outliers for each dependent variable detected through a ROUT method $(Q=2 \%)$ were removed ( $0-2$ samples) prior to correlational analyses. SPSS statistical software (IBM) was 


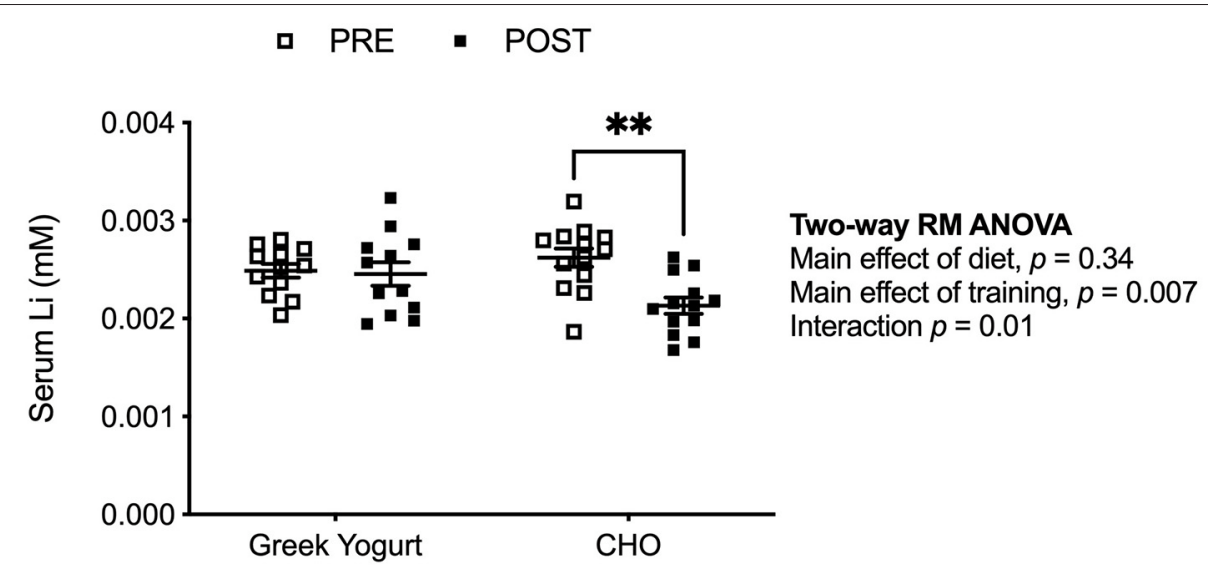

FIGURE 1 | Serum Li concentrations are lowered after exercise training but only in the CHO group and not the GY group. ${ }^{* *} p<0.01$ using a two-way repeated measures ANOVA with a Bonferroni post-hoc test (GY, $n=12$; $\mathrm{CHO}, n=13$ per group).

TABLE 1 | Baseline characteristics of study participants.

\begin{tabular}{lccc}
\hline & CHO $(\boldsymbol{n}=\mathbf{1 3})$ & GY $(\boldsymbol{n}=\mathbf{1 2})$ & $\boldsymbol{p}$ \\
\hline Body mass $(\mathrm{kg})$ & $69.4 \pm 3.1$ & $69.8 \pm 3.0$ & 0.93 \\
Fat-free mass $(\mathrm{kg})$ & $57.6 \pm 2.1$ & $60.4 \pm 2.4$ & 0.39 \\
Fat mass $(\mathrm{kg})$ & $11.8 \pm 1.7$ & $9.3 \pm 1.1$ & 0.25 \\
Body fat $(\%)$ & $16.2 \pm 2.0$ & $13.1 \pm 1.2$ & 0.21 \\
Biceps thickness $(\mathrm{cm})$ & $2.8 \pm 0.1$ & $2.8 \pm 0.1$ & 0.96 \\
Quadriceps thickness $(\mathrm{cm})$ & $3.6 \pm 0.2$ & $3.8 \pm 0.2$ & 0.50 \\
Total strength $(\mathrm{kg})$ & $383.8 \pm 18.7$ & $364.9 \pm 24.5$ & 0.54 \\
P1NP $(\mu \mathrm{g} / \mathrm{L})$ & $115.1 \pm 12.6$ & $101.0 \pm 9.7$ & 0.39 \\
CTX $(\mathrm{ng} / \mathrm{L})$ & $856.8 \pm 78.9$ & $815.0 \pm 65.1$ & 0.69 \\
P1NP:CTX & $0.14 \pm 0.01$ & $0.13 \pm 0.01$ & 0.60 \\
Dietary calcium intake $(\mathrm{mg})$ & $676 \pm 65$ & $698 \pm 75$ & 0.82 \\
Dietary protein intake $(\mathrm{g} / \mathrm{kg})$ & $1.28 \pm 0.07$ & $1.30 \pm 0.09$ & 0.74 \\
Dietary phosphorus intake $(\mathrm{mg})$ & $667 \pm 99$ & $744 \pm 81$ & 0.56 \\
Dietary potassium intake $(\mathrm{mg})$ & $1573 \pm 198$ & $1529 \pm 189$ & 0.87 \\
\hline
\end{tabular}

Values are presented as mean \pm SEM. CTX, $\beta$-isomerized carboxy-terminal cross-linking telopeptides; P1NP, procollagen type $1 \mathrm{~N}$-terminal propeptide. The $p$-value was achieved using a Student's unpaired t-test.

used for all statistical analyses and significance was set at $p \leq 0.05$.

\section{RESULTS}

Li content in Greek yogurt was measured and found to be $0.02 \mathrm{mg} / \mathrm{kg}(0.0025 \mathrm{mM})$ (9). In Figure 1, we examined serum Li concentration pre- and post-exercise training in the GY and CHO groups. Two-way repeated measures ANOVA revealed a significant interaction between diet and training, which indicated that the $\mathrm{CHO}$ group displayed a reduction in serum $\mathrm{Li}$ after training, whereas the GY group did not (Figure 1).
TABLE 2 | Correlational analyses with changes in serum Li (mM) and changes in musculoskeletal variables.

\begin{tabular}{lcll}
\hline & $\boldsymbol{r}$ & $\boldsymbol{p}$ & $\boldsymbol{n}$ \\
\hline$\Delta$ body mass (kg) & 0.11 & 0.59 & 25 \\
$\Delta$ fat-free mass (kg) & 0.26 & 0.20 & 25 \\
$\Delta$ fat mass (kg) & 0.05 & 0.80 & 24 \\
$\Delta$ body fat (\%) & -0.07 & 0.75 & 25 \\
$\Delta$ biceps thickness (cm) & 0.34 & 0.11 & 23 \\
$\Delta$ quadriceps thickness (cm) & -0.02 & 0.91 & 24 \\
$\Delta$ total strength (kg) & 0.57 & 0.004 & 23 \\
$\Delta$ P1NP ( $\mu$ g/L) & 0.20 & 0.34 & 25 \\
$\Delta$ CTX (ng/L) & -0.51 & 0.009 & 25 \\
$\Delta$ P1NP:CTX & 0.52 & 0.008 & 24 \\
\hline
\end{tabular}

CTX, $\beta$-isomerized carboxy-terminal cross-linking telopeptides; P1NP, procollagen type 1 $N$-terminal propeptide.

There were no differences between groups in baseline musculoskeletal variables or dietary intakes of protein, calcium, potassium and phosphorus (Table 1). Changes in serum $\mathrm{Li}$ levels did not correlate with changes in body mass, body composition or muscle size, but a significant positive correlation was observed with changes in total strength (Table 2). While there was no association between changes in serum $\mathrm{Li}$ and changes in P1NP, a significant and negative association between changes in serum $\mathrm{Li}$ and CTX was observed. This led to a significant positive association between changes in serum $\mathrm{Li}$ and the P1NP:CTX ratio. Partial correlational analyses revealed that even after controlling for changes in other dietary dairy components, protein, calcium, phosphorus and potassium, significant associations between changes in serum $\mathrm{Li}$ and total strength and CTX were maintained (Table 3). However, the relationship between changes in serum $\mathrm{Li}$ and the P1NP:CTX ratio was no longer statistically significant. 
TABLE 3 | Partial correlational analyses with changes in serum Li (mM) and changes in total strength and bone turnover markers while controlling for dietary protein, calcium, phosphorus, and potassium intakes.

\begin{tabular}{lrcc}
\hline & $\boldsymbol{r}$ & $\boldsymbol{p}$ & $\boldsymbol{n}$ \\
\hline$\Delta$ total strength (kg) & 0.48 & 0.04 & 23 \\
$\Delta$ CTX (ng/L) & -0.45 & 0.04 & 25 \\
$\Delta$ P1NP:CTX & 0.27 & 0.26 & 24
\end{tabular}

CTX, $\beta$-isomerized carboxy-terminal cross-linking telopeptides; P1NP, procollagen type 1 $N$-terminal propeptide.

\section{DISCUSSION}

In this pilot/exploratory study, we questioned whether GY supplementation would alter serum Li levels after 12 weeks of exercise training, and whether changes in serum Li would correlate with changes in body composition, muscle thickness, total strength and markers of bone turnover in young males. We first demonstrated that serum Li levels were lower with exercise training, and while we do not have an exact explanation for this observation, we suspect that $\mathrm{Li}$ secretion through sweat (i.e., with repeated exercise) could play a role (15). Importantly, the reduction in serum $\mathrm{Li}$ was only observed in the CHO group and not the group supplemented with GY. As dairy products such as yogurt can act as a source of dietary Li (9), this result is not entirely surprising. In fact, post serum Li concentration in the GY group was closely matched with our measured concentration of Li in the actual GY provided to the subjects $(0.0025 \mathrm{mM})$, which is within the range previously reported for dairy products (9). Though we did not measure $\mathrm{Li}$ in the $\mathrm{CHO}$ pudding, we suspect that, given its formulation and composition (2 parts maltodextrin, 1 part chocolate pudding powder and water), levels of $\mathrm{Li}$ would be negligible and possibly even below the level of detection. It is also possible that the $\mathrm{CHO}$ pudding itself may have a serum Li lowering effect or that its consumption replaced other Li-containing foods in the diet; however, this requires further investigation.

In the recent results from the BEAST study intervention, GY supplementation was shown to augment the gains in total strength and FFM and improve bone formation with exercise training $(13,14)$. Though our results showed that changes in serum Li levels did not associate with changes in body composition (i.e., FFM or body fat\%) or muscle size, we did find significant associations between changes in serum Li with changes in total strength and markers of bone turnover. Changes in serum Li positively correlated with total strength even after controlling for other nutrients found in dairy products such as protein, calcium, phosphorus, and potassium. This suggests that those that maintained serum Li exhibited greater strength gains with exercise despite no influence of serum $\mathrm{Li}$ on muscle size or FFM. Nonetheless, this result is consistent with our previous work in rodents, where low dose Li supplementation increased specific force production in the murine soleus and extensor digitorum longus without any apparent changes in muscle mass
(8). With respect to bone turnover, our findings indicate that changes in serum Li may be related to bone resorption, as it was negatively associated with changes in CTX even after controlling for other dietary dairy components. This result is also consistent with our previous rodent work showing that low dose Li supplementation increased the osteoprotegerin:RANKL ratio in the murine femur, which in theory would lead to less osteoclast differentiation/activity (6). When expressed as a ratio, P1NP:CTX provides a biomarker of bone collagen turnover (formation over resorption); and because of Li's negative association with CTX, we also found a significant positive association between changes in serum Li and changes in the P1NP:CTX ratio. However, after controlling for other dietary dairy components such as protein, calcium, phosphorus and potassium, this relationship was no longer significant. This was likely due to other dairy components, namely protein $(r=0.50, p=0.01)$ and calcium $(r=0.45, p$ $=0.03$ ), that were both positively correlated with P1NP:CTX, accounting for some of the independent association with serum Li. This was to be expected as other factors in dairy undoubtedly influence bone turnover $(16,17)$. Of particular importance is dietary protein as it can stimulate insulin-like growth factor 1 expression, which itself enhances bone mineralization and osteoblast differentiation and activation (18-20). In all, while we acknowledge that there are other factors in dairy that contribute to musculoskeletal strength and structure, our findings suggest that dietary Li within dairy may be an additional component worth further exploring.

The overall purpose of this pilot study was to form the basis of further work that will examine the role of dietary Li on musculoskeletal structure and function with and without exercise training. There were some limitations to our present study that should be addressed in the future. First, we could only speculate that the reduction in serum $\mathrm{Li}$ concentration observed after exercise training in the $\mathrm{CHO}$ group occurred through excessive $\mathrm{Li}$ secretion (i.e., sweat). Future studies should specifically measure the amount of Li lost in sweat and urinary secretions. Second, we did not measure the Li concentration in the $\mathrm{CHO}$ pudding, however due to the formulation of it, we expect that the $\mathrm{Li}$ levels would be negligible. Thirdly, participants recorded their food intake for a fewer number of days (3 days) during the 12th week of the intervention compared with baseline (7 days). We also did not control for Li in the dietary record. This is due to limitations in current software used to assess diet records that do not include the capacity to accurately measure Li intake. Nevertheless, a strength of our study was that we measured serum Li concentrations pre- and post-training. The use of muscle biopsies in this study could have provided additional mechanistic insight on the apparent associations between strength gains and serum Li through the examination of fiber type composition, cross-sectional area, single-fiber contractility, and various biochemical signaling pathways. For example, in rodents we have shown that low dose Li supplementation augments specific force production by inhibiting an enzyme called glycogen synthase kinase 3 (GSK3) (8), and therefore, future studies could also examine GSK3 activation in muscle biopsies with dietary Li supplementation. Though the underlying mechanism behind the apparent relationship between strength 
and $\mathrm{Li}$ in rodents and humans remains unknown, in vitro, we have shown that Li enhances myoblast fusion (21), which could enhance the quality of muscle leading to increased force production. Finally and importantly, future studies should also examine whether biological sex plays a potential mediating role as our study is limited by only examining young healthy males.

In conclusion, our findings from our secondary analyses show that exercise training lowers serum Li levels, but GY supplementation can rescue the losses. Interestingly, maintaining and/or elevating serum Li during exercise training is associated with greater gains in total strength and reductions in bone resorption. Since controlling for factors in GY such as protein and calcium weakened these associations, future studies should determine the specific effects of dietary $\mathrm{Li}$ alone on musculoskeletal structure and function.

\section{DATA AVAILABILITY STATEMENT}

The raw data supporting the conclusions of this article will be made available by the authors, without undue reservation.

\section{ETHICS STATEMENT}

The studies involving human participants were reviewed and approved by Brock University Biosciences Research Ethics Board. The patients/participants provided their written informed consent to participate in this study.

\section{REFERENCES}

1. Zhang J, Anshul F, Malhotra DK, Jaume J, Dworkin LD, Gong R. Microdose lithium protects against pancreatic islet destruction and renal impairment in streptozotocin-elicited diabetes. Antioxidants (Basel). (2021) 10:138. doi: $10.3390 /$ antiox 10010138

2. Donaldson IM, Cuningham J. Persisting neurologic sequelae of lithium carbonate therapy. Arch Neurol. (1983) 40:747-51. doi: 10.1001/archneur.1983.0405011 0065011

3. Malhi GS, Berk M. Is the safety of lithium no longer in the balance? Lancet. (2012) 379:690-2. doi: 10.1016/S0140-6736(11) 61703-0

4. Girardi P, Brugnoli R, Manfredi G, Sani G. Lithium in bipolar disorder: optimizing therapy using prolonged-release formulations. Drugs R D. (2016) 16:293-302. doi: 10.1007/s40268-016-0139-7

5. Gitlin M. Lithium side effects and toxicity: prevalence and management strategies. Int J Bipolar Disord. (2016) 4:27. doi: 10.1186/s40345-016-0068-y

6. Kurgan N, Bott KN, Helmeczi WE, Roy BD, Brindle ID, Klentrou P, et al. Low dose lithium supplementation activates Wnt/beta-catenin signalling and increases bone OPG/RANKL ratio in mice. Biochem Biophys Res Commun. (2019) 511:394-7. doi: 10.1016/j.bbrc.2019.02.066

7. Choi SE, Jang HJ, Kang Y, Jung JG, Han SJ, Kim HJ, et al. Atherosclerosis induced by a high-fat diet is alleviated by lithium chloride via reduction of VCAM expression in ApoE-deficient mice. Vascul Pharmacol. (2010) 53:26472. doi: 10.1016/j.vph.2010.09.004

8. Whitley KC, Hamstra SI, Baranowski RW, Watson CJF, Macpherson REK, Macneil AJ, et al. GSK3 inhibition with low dose lithium supplementation augments murine muscle fatigue resistance and specific force production. Physiol Rep. (2020) 8:e14517. doi: 10.14814/phy 2.14517

\section{AUTHOR CONTRIBUTIONS}

RB and LS: data curation, formal analysis, methodology, investigation, validation, writing - original draft, and writingreview and editing. AJ and VF: conceptualization, methodology, investigation, data curation, project administration, resources, formal analysis, validation, and writing-review and editing. All authors contributed to the article and approved the submitted version.

\section{FUNDING}

This work was funded through unrestricted research grants from Brock University to AJ and VF. RB was supported by a Match of Minds Scholarship from Brock University and LS was supported by a Canadian Institutes of Health Research Postdoctoral Fellowship (\#164711). VF was supported by a Canada Research Chair (Tier II) in Tissue Plasticity and Remodeling Throughout the Lifespan. The publication of this study was supported by a Brock University Library Open Access Fund.

\section{ACKNOWLEDGMENTS}

We thank A. Bridge, J. Brown, M. Nasato, and H. Snider for carrying out the original BEAST study intervention and collecting the data. Greek yogurt was kindly provided by Danone Inc. We also thank Liqun Qiu for her assistance with the ICP-MS.
9. Nabrzyski M, Gajewska R. Content of strontium, lithium and calcium in selected milk products and in some marine smoked fish. Nahrung. (2002) 46:204-8. doi: 10.1002/1521-3803(20020501)46:3<204::AID-FOOD204>3.0. $\mathrm{CO} ; 2-8$

10. Schrauzer GN. Lithium: occurrence, dietary intakes, nutritional essentiality. J Am Coll Nutr. (2002) 21:14-21. doi: 10.1080/07315724.2002.107 19188

11. Kapusta ND, Mossaheb N, Etzersdorfer E, Hlavin G, Thau K, Willeit M, et al. Lithium in drinking water and suicide mortality. Br J Psychiatry. (2011) 198:346-50. doi: 10.1192/bjp.bp.110.091041

12. Szklarska D, Rzymski P. Is lithium a micronutrient? from biological activity and epidemiological observation to food fortification. Biol Trace Elem Res. (2019) 189:18-27. doi: 10.1007/s12011-018-1455-2

13. Bridge A, Brown J, Snider H, Nasato M, Ward WE, Roy BD, et al. Greek yogurt and 12 weeks of exercise training on strength, muscle thickness and body composition in lean, untrained, university-aged males. Front Nutr. (2019) 6:55. doi: 10.3389/fnut.2019.00055

14. Bridge AD, Brown J, Snider H, Ward WE, Roy BD, Josse AR. Consumption of Greek yogurt during 12 weeks of high-impact loading exercise increases bone formation in young, adult males-a secondary analysis from a randomized trial. Appl Physiol Nutr Metab. (2020) 45:91-100. doi: 10.1139/apnm-201 9-0396

15. Jefferson JW, Greist JH, Clagnaz PJ, Eischens RR, Marten WC, Evenson MA. Effect of strenuous exercise on serum lithium level in man. Am J Psychiatry. (1982) 139:1593-5. doi: 10.1176/ajp.139.12.1593

16. Tunick MH, Van Hekken DL. Dairy products and health: recent insights. J Agric Food Chem. (2015) 63:9381-8. doi: 10.1021/jf50 42454

17. Ratajczak AE, Zawada A, Rychter AM, Dobrowolska A, KrelaKazmierczak I. Milk and dairy products: good or bad for human bone? practical dietary recommendations for the prevention and 
management of osteoporosis. Nutrients. (2021) 13:1329. doi: 10.3390/nu130 41329

18. Bourrin S, Ammann P, Bonjour JP, Rizzoli R. Dietary protein restriction lowers plasma insulin-like growth factor I (IGF-I), impairs cortical bone formation, and induces osteoblastic resistance to IGF-I in adult female rats. Endocrinology. (2000) 141:3149-55. doi: 10.1210/endo.141. 9.7633

19. Crane JL, Zhao L, Frye JS, Xian L, Qiu T, Cao X. IGF-1 Signaling is essential for differentiation of mesenchymal stem cells for peak bone mass. Bone Res. (2013) 1:186-94. doi: 10.4248/BR2013 02007

20. Bonjour JP. The dietary protein, IGF-I, skeletal health axis. Horm Mol Biol Clin Investig. (2016) 28:39-53. doi: 10.1515/hmbci-2016-0003

21. Kurgan N, Whitley KC, Maddalena LA, Moradi F, Stoikos J, Hamstra SI, et al. A low-therapeutic dose of lithium inhibits GSK3 and enhances myoblast fusion in C2C12 cells. Cells. (2019) 8:1340. doi: 10.3390/cells 8111340

Conflict of Interest: AJ reports grant funding from Dairy Farmers of Canada, Dairy Management Inc., non-financial support from Danone and Parmalat, and consultant and speaker fees from Dairy Farmers of Canada. LS reports grant funding from Dairy Farmers of Canada and salary support from Dairy Management Inc.

The remaining authors declare that the research was conducted in the absence of any commercial or financial relationships that could be construed as a potential conflict of interest.

Publisher's Note: All claims expressed in this article are solely those of the authors and do not necessarily represent those of their affiliated organizations, or those of the publisher, the editors and the reviewers. Any product that may be evaluated in this article, or claim that may be made by its manufacturer, is not guaranteed or endorsed by the publisher.

Copyright (C) 2021 Baranowski, Skelly, Josse and Fajardo. This is an open-access article distributed under the terms of the Creative Commons Attribution License (CC $B Y)$. The use, distribution or reproduction in other forums is permitted, provided the original author(s) and the copyright owner(s) are credited and that the original publication in this journal is cited, in accordance with accepted academic practice. No use, distribution or reproduction is permitted which does not comply with these terms. 